\title{
Theoretical and Experimental Aspects Regarding Nonlinear Effects of Dry Friction and Unbalanced Rotational Mass in a Dynamical System
}

\author{
Stelian ALACI*, Carmen BUJOREANU**, Florina Carmen CIORNEI***, \\ Daniel FODORCAN $* * * *$ \\ *,Stefan cel Mare” University, 13 University Str., Suceava, Romania; E-mail: florina@fim.usv.ro \\ **,,Gheorghe Asachi”" Technical University, 61-63 Dimitrie Mangeron Bd., Iasi, Romania; E-mail: cbujorea@tuiasi.ro \\ ***,,Stefan cel Mare” University, 13 University Str., Suceava, Romania; E-mail: alaci@fim.usv.ro \\ ****Space Applications Services, 325 Leuvensesteenweg, 1932 Zaventem, Brussels, Belgium; fodorcandaniel@yahoo.com \\ crossref http://dx.doi.org/10.5755/j01.mech.24.6.22470
}

\section{Introduction}

Gaudi's affirmation that ,there are no straight lines or sharp corners in nature" [1-2], is one of the most direct and succinct expression of the fact that the world we are living in is a nonlinear one. The development of mathematics is known starting 5000 years ago with the Assyrians, but only relatively recently, ideas such as replacing the arch of a curve, in the vicinity of a point from the curve, with a fragment of the tangent passing through the considered point, were accepted. This lead to the independent invention, by Newton and Leibniz, of differential calculus [3], one of the most powerful instruments of investigation of the phenomena of the world. Not long after, Newton stipulates the renowned second law, [4] this being the first differential equation. Thus appears, a new domain of mathematics where the unknowns are the derivatives of functions, appear. Newton, then Bernoulli and other eminent mathematicians offer methods of solving particular cases of differential equations. From the requirement of modeling the surrounding world, the theory of differential equations develops rapidly and nowadays is a selfdetermining branch of mathematics [3], [5]. At the same time with the development of differential equations theory, the conclusion that there are few situations when an analytical solution can be found was reached. Furthermore, major difficulties occur when the differential equation is not a linear combination of its derivatives and thus the most important criterion of classification of differential equations was also set. Newton's second law and the observation made by Gaudi point to the conclusion that it is expected that the equations describing mechanical phenomena are nonlinear differential equations. The particular case when linear differential equations describe a phenomenon is, under a more rigorous analysis, the effect of an approximation, applicable for a narrow domain of investigation. To support this affirmation one can give examples such as: the constant gravitational acceleration, the relation for the curvature of a bended bean etc. [6]. This procedure of linearization of a nonlinear differential equation (NoDE) in the vicinity of a point is a technique frequently met in the study of NoDE. Fidlin [7] analyses the causes producing nonlinear effects in mechanical applications and firstly identifies the dry friction contacts and collisions. Another source of nonlinearities discovered by Fidlin is an unbalanced part in rotation motion, present in all types of crank mechanisms. The importance of the problem is also underlined in a variety of applications [8-13]. The present paper presents a nonlinear dynamic system where both dry friction and a rotating unbalanced part are present. The unknown parameters are identified and the equations of motion are deduced and afterwards numerically integrated. Experimental tests were carried out using a laboratory device and the results are compared to the theoretical ones.

\section{Theoretical model. Obtaining the equations of mo- tion and their integration. Discussions}

A body having the mass $M$ and the moment of inertia with respect to the center of mass $J_{G z}$ performs a rotation around an axis parallel to the central axis of inertia (the distance between the two axes is $\xi$ ), as in Fig. 1 .

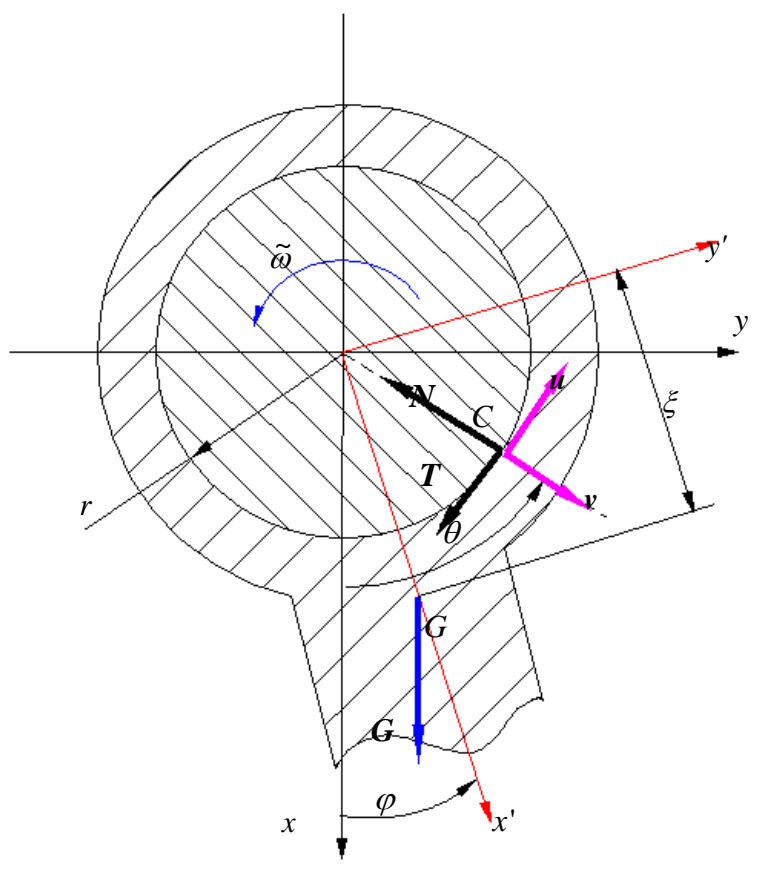

Fig. 1 Eccentric rotor

The rotation pair is obtained using a spindle of $r$ radius and the coefficient of dry friction from the spindlebase contact is $\mu$. The motion of the rotor is desired considering that the initial angular velocity of the rotation motion is $\omega_{0}$. The hypothesis of rigid actuating shaft is accepted and it results that the motion of the rotor is a plan parallel one. Thus, three scalar equations are available: two 
resulting from the theorem of the motion of the center of mass and one from the moment of momentum theorem.

The unknowns of the problem are: the angle $\varphi$ that specifies the position of the center of mass, the values of the normal reaction $\boldsymbol{N}$ and of the friction force $\boldsymbol{T}$ (tangent to the surface of the spindle) with the mention that the Amontons-Coulomb friction law [14] gives the relation between the last two:

$$
T=\mu N
$$

In order to identify the friction force, it must be considered that in the rotation pair there is actually a surface contact and the normal reaction $N$ is in fact the resultant of a system of elementary pressure forces whose reduction conducts to a force $\boldsymbol{N}$ characterized by magnitude and direction. The last unknown is a parameter required to characterize the orientation of the normal reaction; the angle made by the direction of the normal $\mathrm{N}$ with the positive semi-axis $O x$ is chosen to be this parameter. The versor of this direction id denoted $\boldsymbol{u}$ and is expressed as function of the versors $i, j$ and $\boldsymbol{k}$ of the immobile system, using the relations:

$$
\mathbf{u}=\mathbf{i} \cos \theta+\mathbf{j} \sin \theta
$$
defined as:

The versor of the tangent force $\boldsymbol{T}$ denoted $v$ is

$$
\mathbf{v}=\mathbf{k} \times \mathbf{u}=-\mathbf{i} \sin \theta+\mathbf{j} \cos \theta
$$

The versors of the system attached to the rigid, $\boldsymbol{i}^{\prime}$ and $\boldsymbol{j}^{\prime}$ are expressed using similar relations:

$$
\begin{aligned}
& \boldsymbol{i}^{\prime}=\boldsymbol{i} \cos \varphi+\boldsymbol{j} \sin \theta ; \\
& \boldsymbol{j}^{\prime}=-\boldsymbol{i} \sin \varphi+\boldsymbol{j} \cos \theta .
\end{aligned}
$$

The center of mass of the rotor has the position vector expressed by:

$$
\boldsymbol{r}_{G}=\xi \boldsymbol{i}^{\prime}=\xi \boldsymbol{i} \cos \varphi+\xi \boldsymbol{j} \sin \varphi .
$$
is written as:

The theorem of motion of the center of mass [15]

$$
M \ddot{\boldsymbol{r}}_{G}=\boldsymbol{N}+\boldsymbol{T}+\boldsymbol{G},
$$

and explicitly:

$$
M \ddot{\boldsymbol{r}}_{G}=M g \boldsymbol{i}-N \boldsymbol{u}-T \operatorname{sgn} \dot{\varphi} \boldsymbol{v} .
$$

In the relation (8), $\operatorname{sgn} x$ represents the signum function and it was introduced with the purpose to consider the friction force opposing to the relative motion between the contacting surfaces. The moment of momentum theorem [15] written with respect to the center of mass has the following vector form:

$$
J_{G} \ddot{\varphi} \boldsymbol{k}=\overline{G C} \times(-N \boldsymbol{u}-T \operatorname{sgn} \dot{\varphi} \boldsymbol{v}),
$$

where $\overline{G C}$ is the position vector of the point $C$ - the so called point of application of the normal, with respect to the center of mass $G$. From Fig. 1 it is noticed that:

$$
\overline{G C}=-\xi i^{\prime}+r \boldsymbol{u}
$$

Considering all the previous relations and making the calculus, the following system of scalar equations is obtained:

$$
\left\{\begin{aligned}
-M \xi\left(\ddot{\varphi} \sin \varphi+\varphi^{2} \cos \varphi\right)= & M g+\mu M g \operatorname{sgn} \dot{\varphi} \sin \varphi- \\
& -N \cos \varphi, \\
M \xi\left(\ddot{\varphi} \cos \ddot{\varphi}-\dot{\varphi}^{2} \sin \varphi\right)=-\mu N \cos \theta \operatorname{sgn} \dot{\varphi}-N \sin \theta, & \\
J_{z} \ddot{\varphi}= & \{[\mu \cos (\theta-\varphi) \operatorname{sgn} \dot{\varphi}+\sin (\theta-\varphi)] \xi- \\
& -\mu r \operatorname{sgn} \dot{\varphi}\} \mathrm{N} .
\end{aligned}\right.
$$

The system (11) has as unknowns the parameters $\ddot{\varphi}, \theta$ and $N$. The system is linear with respect to the first two unknowns but, considering the angle $\theta$, the solution will be expressed using the function $\operatorname{atan}(x)$, which is a multiform function. To avoid this, two supplementary unknowns, $N_{x}$ and $N_{y}$ are considered:

$$
N_{x}=N \cos \theta ; \quad N_{y}=N \sin \theta .
$$

Now, the system (11) takes the form:

$$
\left\{\begin{array}{l}
-M \xi\left(\ddot{\varphi} \sin \varphi+\dot{\varphi}^{2} \cos \varphi\right)=M g+ \\
\quad+\mu M g \operatorname{sgn} \dot{\varphi} \sin \varphi-N_{x}, \\
M \xi\left(\ddot{\varphi} \cos \ddot{\varphi}-\dot{\varphi}^{2} \sin \varphi\right)=-\mu N_{x} \operatorname{sgn} \dot{\varphi}-N_{y}, \\
J_{z} \ddot{\varphi}=\left[(\cos \varphi+\mu \operatorname{sgn} \dot{\varphi} \sin \varphi) N_{y}+\right. \\
\left.\quad+(\mu \operatorname{sgn} \dot{\varphi} \cos \varphi-\sin \varphi) N_{x}\right] \xi+\mu r \sqrt{N_{x}^{2}+N_{y}^{2}} \operatorname{sgn} \dot{\varphi} .
\end{array}\right.
$$

The first two equations of the system (13) are linear with respect to $N_{x}$ and $N_{y}$. After finding $N_{x}$ and $N_{y}$ from the first two equations and replacing them into the last equation, the following results:

$$
\begin{aligned}
& \left(J_{z}+M \xi^{2}\right) \ddot{\varphi}+M \xi g \sin \varphi+ \\
& +\frac{\mu r M}{\sqrt{1+\mu^{2}}} \sqrt{(g \sin \varphi+\xi \ddot{\varphi})^{2}+\left(g \cos \varphi+\xi \dot{\varphi}^{2}\right)^{2}} \operatorname{sgn} \dot{\varphi}=0 .
\end{aligned}
$$

The Eq. (14) is an irrational equation with respect to $\ddot{\varphi}$ [16]. To solve it, the radical term is passed into the right member and the equation is raised to the power of two and an equation of second degree is obtained. Raising at power introduces strange roots and therefore, after solving the equation of second degree the solution must be chosen by direct verification. The next notations are introduced: 


$$
\begin{aligned}
& A=\left(J_{z}+\xi^{2} M\right)-\mu^{2} r^{2} M^{2} \xi^{2} /\left(1+\mu^{2}\right), \\
& B=\left(J_{z}+\xi^{2} M\right) M \xi g \sin \varphi-\mu^{2} r^{2} M^{2} \xi g \sin \varphi /\left(1+\mu^{2}\right), \\
& C=M^{2} \xi^{2} g^{2} \sin ^{2} \varphi-\mu^{2} r^{2} M^{2} \frac{g^{2}+2 \xi g \dot{\varphi}^{2} \cos \varphi+\xi^{2} \dot{\varphi}^{2}}{1+\mu^{2}}
\end{aligned}
$$

and the solution of equation (14) is one of the solutions of the equation:

$$
A \ddot{\varphi}^{2}+2 B \ddot{\varphi}+C=0,
$$

having the well-known solutions:

$$
\ddot{\varphi}_{1,2}=\left[-B \pm \sqrt{B^{2}-A C}\right] / A .
$$

It is obvious that by replacing the relations (15) into the solutions (17), two nonlinear ordinary differential equations of second order are obtained. The attempt to apply for a given case, a numerical procedure for obtaining the solutions of the two equations, lead to disappointment each time. Practically, the algorithm stopped for values of the integration interval smaller than $1 \mathrm{sec}$, which contradicts the physical reality. A more detailed analysis of the results underlines the correct solution of the equation (16) as:

$$
\ddot{\varphi}_{1,2}=\left[-B-\sqrt{B^{2}-A C} \operatorname{sgn} \dot{\varphi}\right] / A \text {. }
$$

The error was caused by raising the equation (14) to the power of two when:

$$
\operatorname{sgn}^{2} x=1
$$

relation that obviously is not reciprocal. With the law of variation of the position angle $\varphi$ known, the components $N_{x}$ and $N_{y}$ of the normal reaction are found from the first two equations of the system (13):

$$
\begin{aligned}
N_{x}= & \frac{-\ddot{\varphi} \cos \varphi+\dot{\varphi}^{2} \sin \varphi}{1+\mu^{2}} \mu \xi M \operatorname{sgn} \dot{\varphi}+ \\
& +\frac{g+\xi \ddot{\varphi} \sin \varphi+\xi \dot{\varphi}^{2} \cos \varphi}{1+\mu^{2}} M ; \\
N_{y}= & -\frac{\xi \ddot{\varphi} \sin \varphi+\xi \dot{\varphi}^{2} \cos \varphi+g}{1+\mu^{2}} \mu M \operatorname{sgn} \dot{\varphi}+ \\
& +\frac{\dot{\varphi}^{2} \sin \varphi-\ddot{\varphi} \cos \varphi}{1+\mu^{2}} M \xi,
\end{aligned}
$$

and afterwards, the angle $\theta$ is obtained:

$$
\theta=\operatorname{angle}\left(N_{x}, N_{y}\right)
$$

The $\operatorname{angle}(x, y)$ function is the function that returns the angle form the domain $[0,2 \pi)$ made by the vector radius of the point of coordinates $(x, y)$ with the positive $O x$ semi-axis. For a set of values of the parameters from the coefficients $A, B$ and $C$ from relations (15) convenient- ly chosen, the equation (18) was integrated numerically using the Runge-Kutta 4, [17] with constant step-size method. Afterwards, using the equation (21), the angle $\theta$ and the size of the normal reaction $N$ were found. The angular velocities of the rotor and of the direction of normal reaction are presented in Fig. 2. As expected, in a first stage of the motion, the rotor performs a continuous rotation motion, after which it performs an oscillatory motion with linearly decreasing amplitude, characteristic to dry friction. The normal reaction also has a continuous rotation at the beginning of the motion and at a certain instant, it turns into oscillation motion. It is noted that the moments when the two rotation motions change into oscillation motions doesn't coincide, but at the instant of the transformation, both motions have the highest amplitude of the entire process.

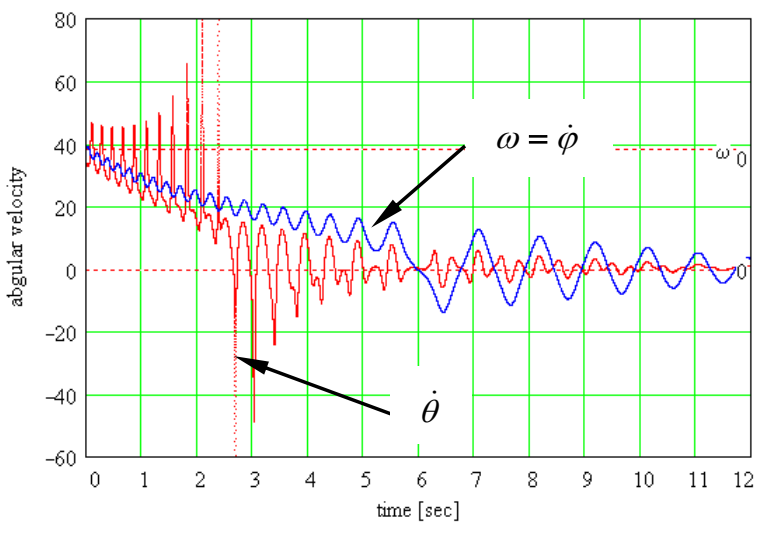

Fig. 2 Angular velocity of the rotor (blue) and angular velocity of the direction of normal reaction (red)

The variations of the dimensionless normal reaction (obtained by dividing to the weight of the rotor) and of the dimensionless angular velocity of the direction of normal reaction (obtained by dividing to the initial angular velocity $\omega_{0}$ ) are presented in Fig. 3 . An interesting conclusion results from the plot: the size of the normal reaction is minimum at the moment when the motion of the normal turns from rotation to oscillation motion.

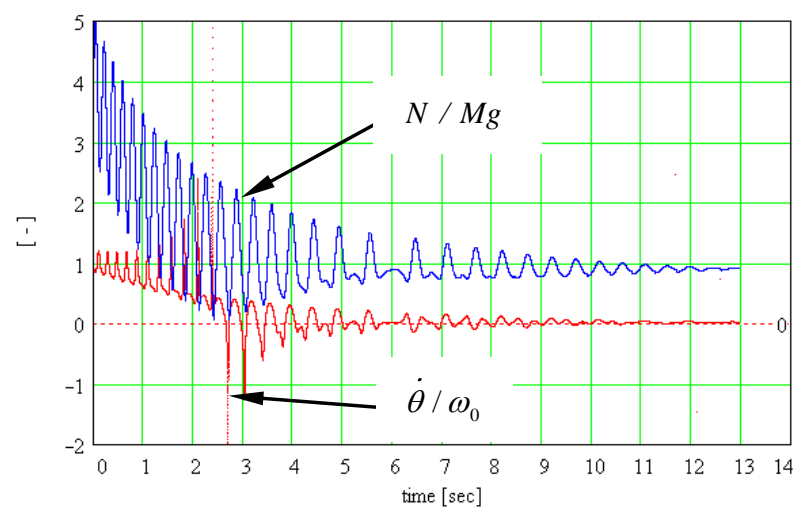

Fig. 3 Dimensionless variation of size of the normal reaction and of the angular velocity of its direction

\section{Experimental device: description, running and re- sults}

A simple device, of schematic shown in Fig. 4, is proposed to carry out tests in order to validate experimen- 
tally the theoretical results obtained above. A steel disc of radius $r_{2}$, mass $m_{2}$ and central moment of inertia $J_{G 2}$ is mounted coaxially to a brass bushing of negligible mass, into which a cylindrical hole of radius $r$ is made. Two holes, diametrically opposed are made into the disc, with the centers at a distance $r_{0}$ from the axis of the disc. Two discs of mass $m_{1}$, radius $r_{1}$ and central moment of inertia $J_{G 1}$ are mounted with screws into the two holes, as shown in Fig. 4: first, both into the same hole to obtain an unbalanced rotor, as in Fig. 4a, and secondly, as in Fig. 4, b, corresponding to a symmetric rotor.

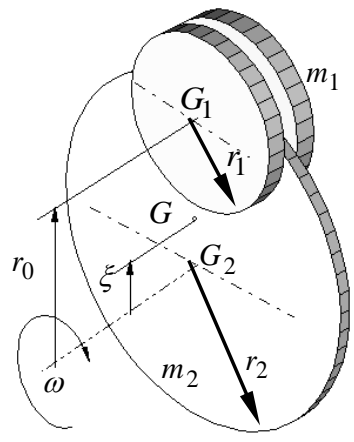

a

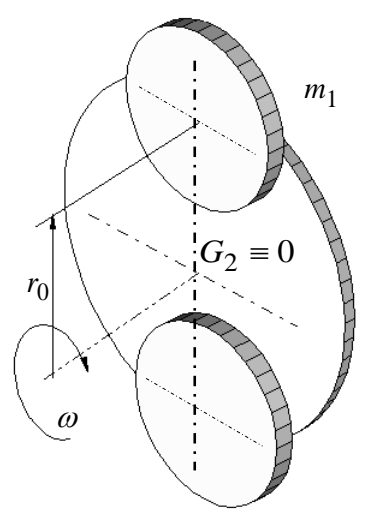

b
Fig. 4 The principle of obtaining the balanced rotor (a) and the unbalanced rotor (b)

The entire assembly can rotate about a rigid steel shaft, mounted horizontally on the working table, as shown in Fig. 5, a, where the general view of the experimental set-up is presented.

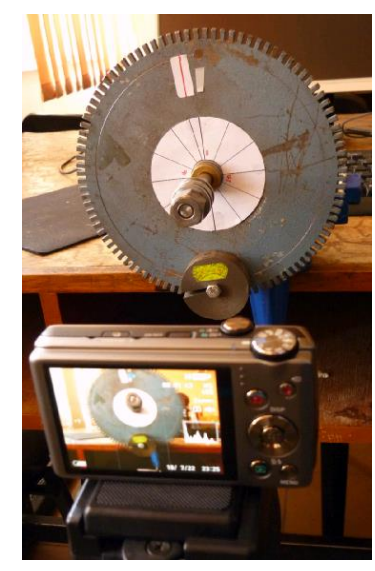

$\mathrm{a}$

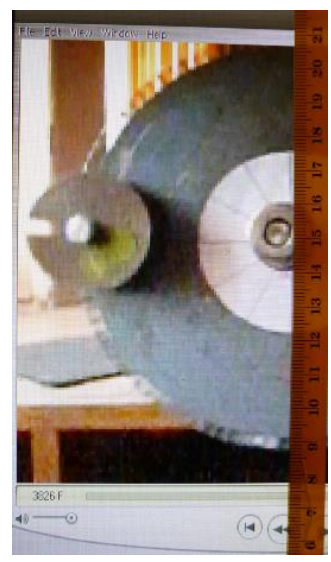

b
Fig. 5 Experimental set-up: (a) general view; (b) detail for finding the law of motion from table data

For the unbalanced rotor as in Fig. 4a, the following characteristics are found:

- the mass $M$ :

$$
M=m_{2}+2 m_{1}
$$

- the position of the center of mass with respect to Oxy frame:

$$
\xi=2 m_{1} r_{0} /\left(2 m_{1}+m_{2}\right)
$$

- the moment of inertia with respect to the center of mass:

$$
J_{z}=J_{G 2}+m_{2} \xi^{2}+2\left[J_{G 1}+2\left(r_{0}-\xi\right)^{2}\right]
$$

For the balanced rotor, the mass is found using the relation (22), the center of mass is coincident with the center of the disc and the moment of inertia is:

$$
J_{z}=J_{G 2}+2\left(J_{G_{1}}+m_{1} r_{0}^{2}\right)
$$

Table 1 presents the constructive and inertial characteristics of the rotor in a concise manner.

Table 1

Constructive and inertial parameters

\begin{tabular}{|c|c|c|}
\hline Notation & Value & Units \\
\hline$r$ & 0.005 & {$[\mathrm{~m}]$} \\
\hline$r_{0}$ & 0.080 & {$[\mathrm{~m}]$} \\
\hline$m_{1}$ & 0.300 & {$[\mathrm{~kg}]$} \\
\hline$r_{1}$ & 0.025 & {$[\mathrm{~m}]$} \\
\hline$J_{G 1}$ & $9.375 \cdot 10^{-5}$ & {$\left[\mathrm{~kg} \cdot \mathrm{m}^{2}\right]$} \\
\hline$m_{2}$ & 1.100 & {$[\mathrm{~kg}]$} \\
\hline$r_{2}$ & 0.095 & {$[\mathrm{~m}]$} \\
\hline$J_{G 2}$ & $4.96 \cdot 10^{-3}$ & {$\left[\mathrm{~kg} \cdot \mathrm{m}^{2}\right]$} \\
\hline$M$ & 1.700 & {$[\mathrm{~kg}]$} \\
\hline$\xi$ & 0.028 & {$[\mathrm{~m}]$} \\
\hline$J_{\text {zunbalanced }}$ & $7.64 \cdot 10^{-3}$ & {$\left[\mathrm{~kg} \cdot \mathrm{m}^{2}\right]$} \\
\hline$J_{\text {zbalanced }}$ & $8.99 \cdot 10^{-3}$ & {$\left[\mathrm{~kg} \cdot \mathrm{m}^{2}\right]$} \\
\hline
\end{tabular}

The motion of the assembly is initiated by hand. In order to establish the motion of the rotor, a disc with twelve marks angularly equidistant is applied on the frontal face of the steel disc. The motion is filmed using a high speed camera that captures 480 frames/sec and afterwards the footage is split into frames. The time required for a radius to change the orientation with a stipulated angular position is found. Initially, a non-contact digital tachometer is utilized for establishing the law of motion. But for short intervals of motion (of order 3-5 sec) and reduced number of rotations, the tachometer does not accurately describe the variation of the signal. regarding the assembly according to the schematics from Fig. 4, b, it is intended to test the hypothesis of constant friction torque accepted in the case of dry friction pairs for the symmetrical rotor; if the assumption is confirmed, it allows for finding the coefficient of sliding friction from the rotation pair, parameter required for the simulation of the asymmetric rotor motion. The experimental data obtained for the two cases proposed in Fig. 4 are presented in Fig. 6. For the unbalanced rotor, the angular step considered for the instants of recording the position of the radius is $60^{\circ}$. For the time interval with oscillatory motion, the position and instant corresponding to convenient positions of the rotor were established. For the balanced rotor, the moments consequent to a series of complete rotations were found. The experimental data for the cases from Fig. 4, presented in graphical form in Fig. 6, confirm that the friction torque is constant for the balanced rotor (the straight line shape of the plot) while for the un- 
balanced rotor, the shape of the plot can be compared to the one from Fig. 2.

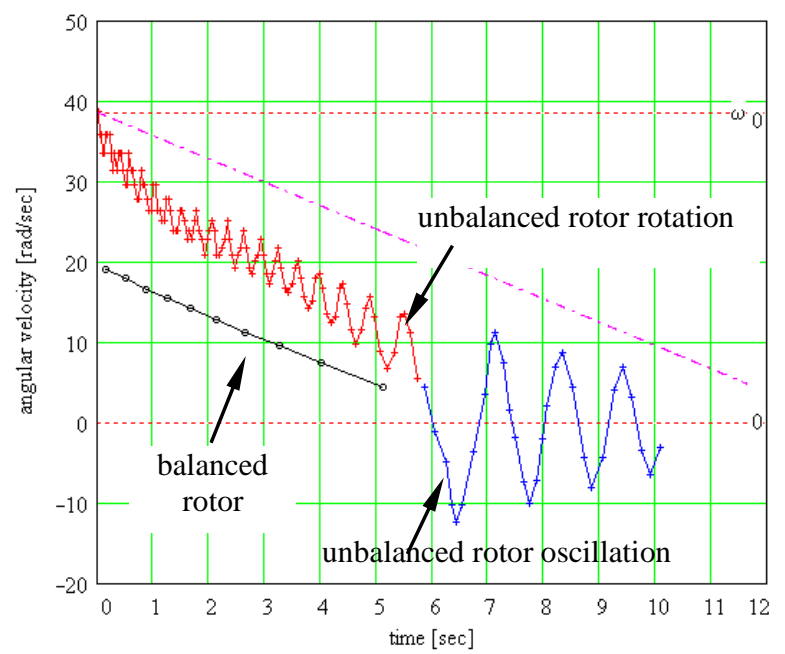

Fig. 6 Experimental data for the symmetric and asymmetric rotor

The moment of momentum theorem written for the balanced rotor is written:

$$
J_{z} \varepsilon=-\mu M g r
$$

resulting in an equation from which the coefficient of sliding friction may be found. The numerical value obtained for the slope of the interpolation line $\varepsilon=-3.008 \mathrm{rad} / \mathrm{sec}$, leads to a value of the coefficient of sliding friction in the bearing $\mu=0.325$ which is in agreement to the values presented in technical literature [14], [18]. Since the initial angular velocity cannot be ensured constant through manual launching, a straight line, parallel to the plot of the symmetric rotor, is traced through the first point of the graph of the unbalanced rotor. This corresponds to the variation of the angular velocity of a symmetrical rotor launched at the same initial velocity as the asymmetrical rotor. Therefore, a comparison can be made between the types of speed variation for the two situations, resulting in the observation that for the eccentric rotor, the angular velocity decreases more rapid than for the balanced rotor.

In Fig. 7 there are presented side by side the experimental data and the theoretical solution for the motion of the unbalanced rotor.

The excellent concordance between experimental and theoretical results for the rotational phase of the motion is noted. During the oscillation phase, a delay appears at a certain time between the model and the experiment; a possible cause may be the change of rotation sense. Thus, a more complex model is necessary for considering both static friction and dynamic friction (the static coefficient of friction being greater than the dynamic coefficient of friction [19]).

Another aspect of practical significance concerns the energy. For a body in rotation motion about a fixed axis, the loss coefficient $\psi$ defined as the ratio between the variation of kinetic energy and the initial kinetic energy, $\Delta E_{c} / E_{C_{0}}$ can be expressed by the relation:

$$
\psi=-\Delta E_{C} / E_{C_{0}}=\left(\omega_{0}^{2}-\omega^{2}\right) / \omega_{0}^{2} .
$$

The evolutions of the loss coefficient for the two solutions from Fig. 4, for the same initial angular velocity, are presented comparatively in Fig. 8.

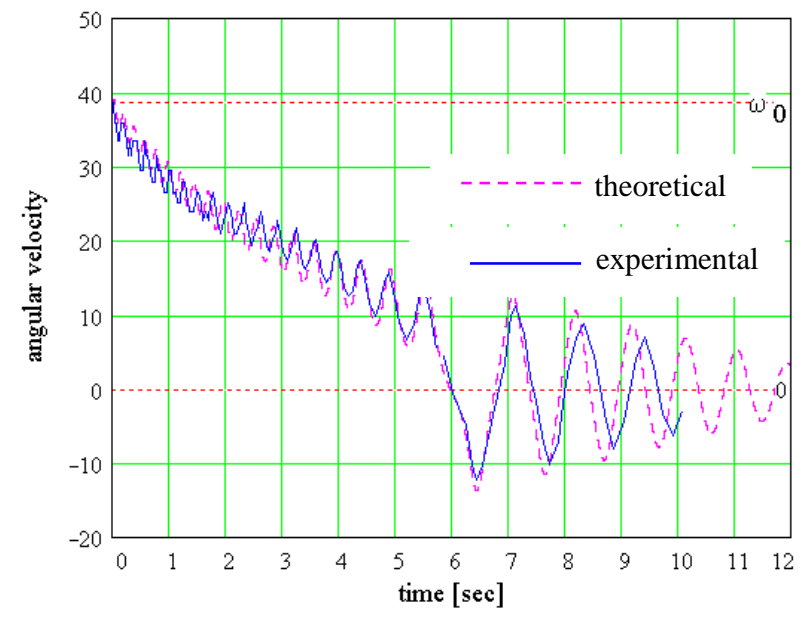

Fig. 7 Theoretical data and experimental results for the motion of the unbalanced rotor

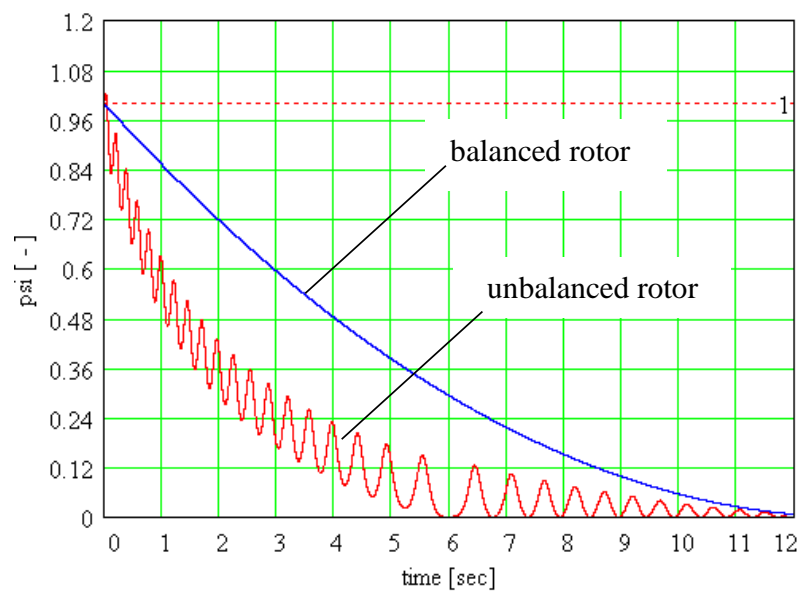

Fig. 8 The loss coefficient for the two types of rotor

Fig. 8 illustrates that for the unbalanced rotor, the energy dissipation is quicker. Using the relation (18) it is proven that the tendency of energy dissipation intensifies significantly with increased launching velocity.

\section{Conclusions}

The paper presents the theoretical and experimental study of an unbalanced rotor, running in dry friction conditions. Both the dry friction and the centrifugal forces induced by the eccentricity of the rotor are sources of nonlinearity of the motion of the rotor.

In the first part of the paper, the unknowns of the problem are identified: the law of motion, the normal force from the pair, and its orientation. The equations of motion are deduced next. In order to obtain the explicit from of the law of motion of the rotor, two new variables are introduced: the projections of the reaction force from the pair on the axis of the fixed system. Thus, the second derivative with respect to the angle of position of the rotor appears in an irrational equation. The explicit form of the differential equation of motion is sought, for which it is necessary to solve the irrational equation, and to choose from the solutions the one that describes the actual motion of the rotor. The nonlinear differential equation of motion is numerical- 
ly integrated for a set of parameters conveniently chosen and it is observed that the motion of the rotor has two stages: first, a continuous rotation, and secondly, an oscillatory motion with linear amplitude attenuation till motionless phase. The same remark can be made concerning the direction of normal reaction from the joint of the rotor, with the mention that the instants when the regime changes differ for the rotor and for the normal reaction, the oscillations of the normal reaction start earlier than the oscillations of the rotor. It must also be mentioned that at the instants when the change from the rotation to oscillation regime happens, the amplitudes of the angular velocity of the rotor and of the direction of normal reaction attain a maximum. In addition, the moment when the regime of motion of the direction of normal reaction is changing coincides to the moment when the value of the reaction attains a minimum.

For the experimental validation of the theoretical results, a simple device is used, consisting in a disc that can rotate in a vertical plane, about a cantilever shaft. Two identical parts can be attached to the disc either in the same position, to obtain an unbalanced rotor, or diametrically opposed, to form a balanced rotor. The rotor is launched into rotation motion by hand, the motion is recorded using a high speed camera (480 frames/sec) and after splitting the footage into frames, the rotation versus time dependency values are displayed in a table.

For the case of the balanced rotor, the decrease of angular velocity is linear, which confirms a constant angular acceleration, resulting from a constant friction torque in the bushing. This allows finding the coefficient of sliding friction from the bushing of the rotor - a parameter required for the theoretical study of the unbalanced rotor. The experimental data lead to the conclusion that the decrease of angular velocity is more rapid for the eccentric rotor than for the balanced rotor.

There is full agreement between experimental and theoretical results and thus the correctness of the theoretical model is proved.

\section{References}

1. Niemann, M. 2015. Constructive Generation Methods for Dungeons. Seminar-Thesis in Procedural Content Generation for Games. Westfalische Wilhelms Universitat Munster. $32 \mathrm{p}$.

2. Lorenzi, M. G.; Francaviglia, M. 2010. Art \& Mathematics in Antoni Gaudi's architecture: "La Sagrada Família", Aplimat-Journal of Applied Mathematics 31: 125-146.

3. Merzbach, U. C.; Boyer, C. B. 2011. A History of Mathematics (3rd ed.), New York: Wiley. 688 p.

4. Newton, I. Translated into English by Motte A. 1846. The Mathematical Principles of Natural Philosophy. Published by Daniel Adee [Accessed 02.05.2018]. http://redlightrobber.com/red/links_pdf/Isaac-NewtonPrincipia-English-1846.pdf.

5. Sasser, J. E. 1992. History of ordinary differential equations: the first hundred years, Proceedings of the Midwest Mathematics History Society. $12 \mathrm{p}$.

6. Frisch-Fay, R. 1962. Flexible bars. London. Butterworths. 228 p.

7. Fidlin, A. 2006. Nonlinear Oscillations in Mechanical Engineering. Berlin Heidelberg: Springer Verlag. 358p.

8. Flores, P.; Lankarani, H. M. 2016. Contact Force
Models for Multibody Dynamics. Springer. $171 \mathrm{p}$.

9. Jonusas, R.; Juzenas, E.; Juzenas, K.; Meslinas, N. 2012. Modelling of rotor dynamics caused by of degrading bearings, Mechanika. 18(4): 438-441.

10. Padgurskas J.; Rukuiža, R.; Bansevičius, R.; Jūrènas, V.; Bubulis, A. 2015. Impact of the tribological characteristics on the dynamics of the ultrasonic piezoelectric motor, Mechanika. 21(1): 51-55.

11. Dzhilavdari, I. Z.; Riznookaya, N. N. 2008. Studies of the dynamics of free microoscillations of a pendulum supported by two balls, Journal of Friction and Wear 29(1): 1-6.

12. Song, Z.; Ma, Z. 2010. Nonlinear vibration analysis of an eccentric rotor with unbalance magnetic pull, IOP Conf. Series: Earth and Environmental Science 12. 012110 http://dx.doi.org/10.1088/1755-1315/12/1/012110.

13. Wan, C. J.; Bernstein, D. S.; Coppola, V. S. 1996. Global stabilization of the oscillating eccentric rotor, Nonlinear Dynamics 10: 49-62.

14. Stachowiak, G. W.; Batchelor, A. W. 2005. Engineering Tribology. Butterworth Heinemann. 832 p.

15. Pytel, A.; Kiusalaas J. 2016. Engineering Mechanics: Dynamics. CL Engineering Publishing. 672 p.

16. Tussy, A. E.; Gustafson, R.D. 2006. Elementary and Intermediate Algebra, Updated Media Edition. Cengage Learning. $1176 \mathrm{p}$.

17. Celia, M. A.; Gray, W. G. 1991. Numerical Methods for Differential Equations: Fundamental Concepts for Scientific \& Engineering Appl. Prentice Hall. 464 p.

18. Bhushan, B.; Gupta, B. K. 1991. Handbook of tribology. McGraw Hill. 1168 p.

19. Marques, P. F. L.; Flores, P; Claro, J. C. P.; Lankarani, H. M. 2016. A survey and comparison of several friction force models for dynamic analysis of multibody mechanical systems, Nonlinear Dynamics 86(3): 1407-1443. http://dx.doi.org/10.1007/s11071-016-2999-3.

\section{S. Alaci, C. Bujoreanu, F. C. Ciornei, D. Fodorcan}

\section{THEORETICAL AND EXPERIMENTAL ASPECTS REGARDING NONLINEAR EFFECTS OF DRY FRICTION AND UNBALANCED ROTATIONAL MASS IN A DYNAMICAL SYSTEM}

S u m m a r y

The paper presents a theoretical analysis of a nonlinear system with dry friction and unbalanced rotational mass. The equations of motion are deduced and integrated numerically. Then, the size and orientation of the normal reaction force can be obtained. The model predicts a rotation motion phase followed by an oscillation one. The experimental results obtained using a laboratory set-up are in very good agreement to the predictions of the theoretical model.

Keywords: nonlinear dynamics, dry friction, unbalanced rotor.

Received August 05, 2018

Accepted December 12, 2018 